\title{
Meaning of Headache at the Emergency Department
}

\author{
Onur Incealtin ${ }^{1,2}$ \\ 'Ondokuz Mayıs University Faculty of Medicine Department of Emergency Medicine \\ ${ }^{2}$ Goztepe Prof. Dr. Suleyman Yalcin City Hospital
}

\begin{abstract}
AIM: We investigated the meaning of headache in the emergency room, as a precursor symptom of structural or systemic disorders, especially life-threatening neurological disorders, or as one of the other headaches unrelated to structural disorders in patients who applied to the emergency department with headache complaints. The causes of primary and secondary headaches should be carefully differentiated from each other at the emergency situations.

METHODS: Patients aged 18 years and older, who applied to the Emergency Department of the Faculty of Medicine of 19 Mayis University, with a complaint of headache were included in the study. Primary headaches were divided into subtypes according to the IHS (International Headache Society) classification. Secondary headaches were divided into two subgroups as neurological and nonneurological.

RESULTS: 200 patients, 112 women and 88 men, who applied to the emergency department with the complaint of headache, were included in the study. Migraine was found in 48 patients, tension-type headache in 29 patients, cerebrovascular disease in 52 patients, CNS infection in 4 patients, intracranial mass in 11 patients, traumatic bleeding in 6 patients, psychiatric disorders in 8 patients, sinusitis in 9 patients, and metabolic disease in 22 patients. Cranial CT was normal in 10 patients with primary headache, cerebral hemorrhage in one patient and cerebral edema was found in one patient. Cranial CT was normal in 18 patients with secondary headache, SAH in 40 patients, intracerebral hemorrhage in 4 patients, intracranial mass in 10 patients, subdural hematoma in 4 patients, cerebral edema and ischemia in 6 patients, and epidural hematoma were found in 1 patient.
\end{abstract}

CONCLUSION: The causes of patients presenting to the emergency department with headache were cerebrovascular disease, migraine, and tension-type headache. In our study, the most common cause was cerebrovascular disease. The prevalence of migraine varies between $3.4-6.1 \%$ in men and $12.9-17.7 \%$ in women. In our study, the incidence of migraine was $23 \%$, and it was seen 3 times more in women than in men.

Keywords: Emergency Department, Primary Headache, Secondary Headache, Migraine

\section{Introduction and Purpose}

Headache is one of the most common complaints in society. Almost all of the society (96\%) complains of a headache at some point in their life $(1,2)$. In this study, we investigated the meaning of headache in the emergency department, as a precursor symptom of structural or systemic disorders, especially life-threatening neurological disorders, or as one of the other headaches unrelated to structural disorders, in cases who applied to the emergency department with headache complaints. The main causes of headache at the emergency room are cerebrovascular disorders, migraine and tensiontype headache, respectively $(3,4)$. Headaches differ significantly with respect to pain power, severity, location and frequency. Primary headache disorders include migraine, tension-type headache, cluster headache, and various headaches not associated with structural disorders (5). Migraine prevalence at the emergency room changed from $15 \%$ to $32 \%$ in the literature $(6,7)$.

In secondary headaches, headache appears as a symptom of another organic disorder that can be detected (5). Rarely, a secondary headache might be a sign of a severe underlying medical illness such as brain infection, brain tumor or hydrocephalus. Every patient with headache at the emergency room should be examined carefully if it is a secondary headache or not. Because, some causes of secondary headaches imitate like primary headaches, and increase the hospitalization time and collateral damages $(8,9)$. 
The main role of emergency services in the investigation of headache is the recognition of secondary headaches, especially neurological ones. Prospective studies examining the frequency of headaches in emergency departments are extremely sparse $(1,3)$.

In the diagnosis and classification of headache, the classification and diagnostic criteria created by the International Headache Society in 2004 are used (9).

The first scientific study on migraine was made by Edward Liveing in 1873 (9). It has been stated that there is a close relation ship between migraine and epilepsy, and both are caused by discharges from the central nervous system, and this is attributed to disorders in the autonomic nervous system. This idea is very important because it is a new view different from the vascular approach (RASKIN88). In 1900, Degl stated that menstrual migraine and other migraine types occur when the swelling that occurs from time to time in the pituitary gland presses on the trigeminal nerve. Möllendorf first described cluster headache in 1913 (10). The International Headache Committee made the latest classification in 1988. The second edition took place at the beginning of 2004 (11). Many questions about headache have not yet been answered.

\section{Methods and Materials}

This prospective study was conducted on patients aged 18 years and older who applied to the Emergency Department of Ondokuz May1s University Faculty of Medicine between July 2004 and December 2005 with the complaint of headache, after the approval of the ethics committee.

Inclusion criteria:

1. Age group 18 years and older

2. Headache being the main complaint

3. Diagnosis and treatment in the emergency room Exclusion criteria:

1. Age group under 18 years old

2. Other symptoms as prominent as the headache

3. Differential diagnoses or treatment in other centers

The age, gender, occupation, characteristics of the headache, medications used, information about the history, family history, physical and neurological examination findings and laboratory findings of the patients were recorded on the special forms created for the study.

\section{Analysis of Data}

The genders were compared in terms of the incidence of primary and secondary headaches. The incidence of migraine and tension headaches between men and women was compared.

Age groups were compared according to the incidence of primary and secondary headaches. Age groups, primary and secondary headaches were compared according to the frequency of $\mathrm{CT}$ findings.

Migraine and tension headache cases were compared according to the frequency of tomography findings. The data were loaded into the SPSS (Statistical Package for Social Science) computer program. Chi-square test was used for cross-table comparison. The Student-T Test was used for data with normal variables to compare the means, and the ManWhitney-U test was used to compare the pairwise means that did not show normal distribution. After analysis of variance, Tukey and Bunferroni multiple comparison tests were planned to be used for multiple comparisons of the means. $p<0.05$ values were considered significant.

\section{Results}

Patients aged 18 years and older who applied to the Emergency Department of the Faculty of Medicine of 19 Mayıs University, with a complaint of headache were included in the study. Those diagnosed with primary headache were treated medically and neurology outpatient control was recommended. For those diagnosed with secondary headache, consultation was requested from the relevant departments. Primary headaches were divided into subtypes according to the IHS (International Headeche Society) classification. Secondary headaches were divided into two subgroups as neurological and non-neurological.

A total of 200 patients, 112 women and 88 men, who applied to the emergency department with the complaint of headache, were included in the study. 76 patients had primary headache and 124 patients had secondary headache (Figure 1).

As the cause of headache; migraine was found in 48 patients, general headache in 29 patients, cerebrovascular disease in 52 patients, CNS infection in 4 patients, intracranial mass in 11 patients, traumatic bleeding in 6 patients, psychiatric disorders in 8 patients, sinusitis in 9 patients, and metabolic disease in 22 patients 
A

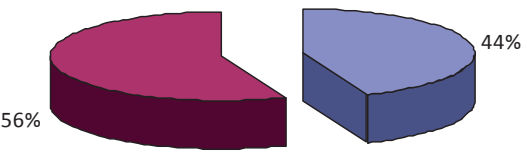

B

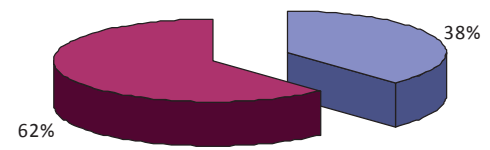

C

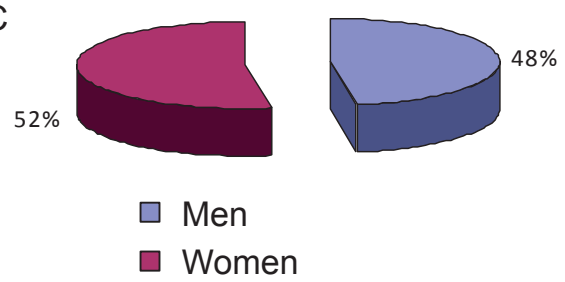

Figure 1. A- $56 \%$ of patients were woman, who applied with headache to emergency room. Primary (B) and secondary (C) headache ratio was found in women as $62 \%$ and $52 \%$, respectively.

(Figure 2). We found the main cause of headaches in our emergency department was cerebrovascular disease.

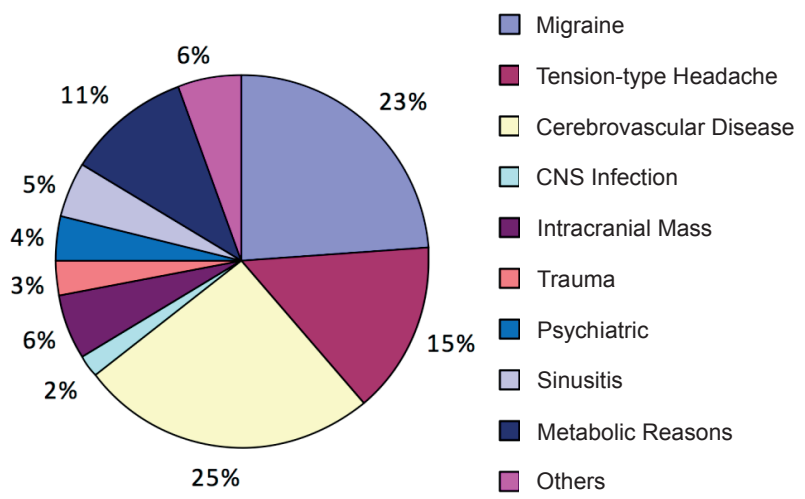

Figure 2. Causes of headaches in emergency room.

Cranial CT was performed in 12 patients with primary headache and 83 patients with secondary headache. Cranial CT was found to be normal in 10 patients with primary headache, cerebral hemorrhage was found in one patient and cerebral edema was found in one patient. Cranial CT was normal in 18 patients with secondary headache, $\mathrm{SAH}$ in 40 patients, intracerebral hemorrhage in 4 patients, intracranial mass in 10 patients, subdural hematoma in 4 patients, cerebral edema and ischemia in 6 patients, and epidural hematoma in 1 patient (Figure 3).

Headache is among the most common reasons for admission to the emergency department. While the frequency of headache in the emergency department was between $1 \%$ and $16 \%$, we found the frequency of headache was 3\%. Cerebrovascular disease was found in $25 \%$, migraine in $23 \%$, and general headache in $15 \%$ of the patients who applied with the complaint of headache (Figure $2)$. The reasons for the patients who applied to the emergency department with headache in the studies; Cerebrovascular disease was found in the first place, followed by migraine and tension headache, respectively. In our study, the most common cause was cerebrovascular disease. The prevalence of migraine varies between $3.4-6.1 \%$ in men and $12.9 \%-17.7 \%$ in women. In our study, the incidence of migraine was $23 \%, 3$ times more in women than in men (Figure 4).



Figure 3. Cranial tomography results of patients, who diagnosed with secondary headaches. 83 patients diagnosed with secondary headaches, the main cause was subarachnoid hemorrhage (40 patient).

\section{Discussion and Conclusion}

Headache is among the most common reasons for admission to the emergency department. In studies in the literature, the frequency of headache in the emergency department was found to be between $1-16 \%(1,5)$. In our study, the frequency of headache in the emergency department was found to be $3 \%$.

When the patients who applied to the emergency department with the complaint of headache were examined, $48 \%$ primary and $52 \%$ secondary headaches were observed $(8,12)$. In our study, $38 \%$ of the patients with headache were evaluated as primary and $62 \%$ as secondary headache. The reason for the higher incidence of secondary headache may be that more patients with secondary 


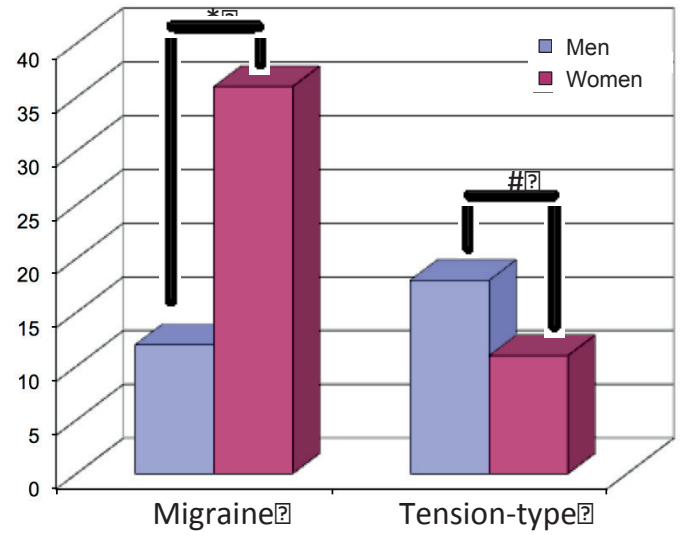

Figure 4. The comparison of migraine and tension-type headaches in genders. 12 men and 36 women were diagnosed with migraine. 18 men and 11 women were diagnosed with tension-type headache. Results were significantly different, $\left({ }^{*}\right)$ and $(\#), p<0,05$.

headaches are referred because our hospital is a tertiary health care institution $(1,8)$.

Cerebrovascular disease was observed in $25 \%$ of our patients who applied with the complaint of headache, migraine in $23 \%$ and tension headache in $15 \%$. Patients with tension headache do not apply to the emergency department as often as patients with migraine, because tension headaches tend to have a slower onset and less severity.

The reason for the higher rate of cerebrovascular disease is that a large number of patients are referred to our hospital because it is a tertiary health center. Migraine and tension headaches constitute 25-55\% of all headaches in the emergency department $(5,7)$. We found the rate of migraine and tension headache to be compatible with the literature.

The frequency of migraine in the emergency department varies between $15-32 \%(7,13)$. In our study, the incidence of migraine was $23 \%$. Migraine is more common in women. In one study, the frequency of migraine was found to be $15-18 \%$ in women and $6 \%$ in men $(7,14)$. The reason for this is thought to be hormonal changes and their effects (15). We found that the frequency of migraine is 3 times higher in women than in men.

Tension-type headache was more common in women than men $(7,16)$. In our study, tension headache was more common in males. This may be due to regional differences. Because studies have reported that the distribution of primary headaches may be affected by social causes (17).

Studies on Brain Tomography in primary headaches are few. Sargent et al found cortical atrophy and old infarcts in $28 \%$ of 177 patients with tension-type headache and migraine on non-contrast $\mathrm{CT}$ that did not affect the clinical course and treatment (10). Carrera et al. obtained normal cranial CT findings in all 53 patients with primary headache who had normal neurological examination (18). In our study, 12 patients with primary headache characteristics had normal findings on tomography in $84 \%$ of these patients, brain edema in $8 \%$, and hemorrhage in $8 \%$. Abnormal findings were observed in only 2 patients. The primary headache diagnosis of these patients was migraine. Migraine family histories were unclear. These results show that primary headaches also require a complete neurological evaluation.

Mills et al. found a high rate of intracranial pathology by applying emergency imaging methods in patients presenting with headache (19). The patients evaluated by imaging methods were coma, trauma patients, patients with epileptic seizures, patients with hemiparesis and patients with headache.

Sudden onset of headache is among the warning criteria for secondary headaches $(5,8,20)$. Diagnoses with high mortality, such as cerebrovascular disease, were observed more frequently in our patients with sudden onset headaches. While $77 \%$ of our patients who applied to our emergency department with a sudden onset headache complaint were secondary headaches, $23 \%$ had primary headaches.

Aygün et al. found cerebrovascular disease in 71\% of patients with sudden onset headache (3). These results show that the sudden onset of headaches is an important warning criterion for secondary headaches (3). Although sudden onset headaches are likely to be secondary, other warning criteria of secondary headache should be sought in slowonset cases. Because it has been reported that the pain character of some tumors is compatible with tension headache. Headache was due to secondary causes in approximately half of the patients with slow-onset headache in our country. Most of these causes were intracranial masses (21).

In the studies, $82 \%$ of the cases with primary headache and $43 \%$ of the cases with secondary headache were in the young age group. While there was no patient over 65 years of age among the primary headache cases, the patients in this age group comprised $19 \%$ of the patients with secondary headache. The rate of pathological findings in the CT of the cases was significantly higher in the group over 65 years of age $(22,23)$. In our study, 
the incidence of secondary headache increased with increasing age. This finding shows that in patients with headache that starts at an advanced age, it is necessary to be careful in terms of secondary headache. Primary headaches are more common in middle age, and their frequency decreases after the age of 40 (15). In another study, it was observed that the prevalence of headache decreased with advancing age. We did not observe any patient over 65 years of age with primary headache.

In the studies performed, the causes were observed in the cases, who applied to the emergency department with headache, cerebrovascular disease $(25 \%)$, migraine $(23 \%)$ and tension headache $(15 \%)$, respectively $(24,25)$. We found the most common cerebrovascular disease in patients presenting with headache. In these results, we thought that referral of more patients to the emergency department of our hospital from other health institutions was effective.

Headache is quite common in the emergency room. Cases presenting to the emergency department with headache should be carefully examined and it should be ensured whether there is a secondary headache. Because, sometimes secondary headaches may exhibit the characteristics of primary headaches. For this purpose, warning criteria for secondary headache should be sought carefully (8). In addition, it should always be kept in mind that headache is important in cases presenting with headache in emergency conditions.

\title{
References
}

1. Kelly AM, Kuan WS, Chu KH, Kinnear FB, Keijzers G, Karamercan MA, Klim S, Wijeratne T, Kamona S, Graham CA, Body R, Roberts T, Horner D, Laribi S; and the HEAD Study Group. Epidemiology, investigation, management, and outcome of headache in emergency departments (HEAD study)- A multinational observational study. Headache. 2021 Nov 2. doi: 10.1111/head.14230

2. Dawson JA. Your questions answered migrain and other headaches. Churchill Livingstone; London 2003.

3. Aygun,D, Bildik F. Clinical warning criteria in evaluation by computed tomography the secondary neurological headaches in adults. Eur J of Neurol 2003; 10: 437-442. https://doi.org/10.1046/j.1468-1331.2003.00645.x

4. Bobker SM, Safdieh JE. Approach to Headache. Semin Neurol. 2021 Dec; 41(6): 633-643. doi:10.1055/s-0041-1726360.

5. Newman LC, Lipton RB. Emergency department evalutiopn of headache. Neurol Clin 1988; 16: 285-303

6. Ng JY, Hanna C. Headache and migraine clinical practice guidelines: a systematic review and assessment of complementary and alternative medicine recommendations. BMC Complement Med Ther. 2021 Sep 22; 21(1): 236.

7. Neumeier MS, Pohl H, Sandor PS, Gut H, Merki-Feld GS, Andrée C. Dealing with Headache: Sex Differences in the Burden of Migraine- and Tension-Type Headache. Brain Sci. 2021 Oct 5; 11(10): 1323.

8. Zhu K, Born DW, Dilli E. Secondary Headache: Current Update. Headache. 2020 Nov;60(10): 2654-2664.

9. Rasmussen BK, Jensen R, Scrholl M. Epidemiology of headache in a genel population a prevelance study.J Clin Epidemiol 1991; 44: 1147-1157

10. Sargent DJ, Solbach P. Medical evalution of migrainosus : rewiew of the value of labarotary and radiologic tests. Headache 1983; 23: 62-65

11. Silberstein SD, Lipton RB, Guadsby P. In:.Clinics Practice Martin Denitz. Headache London 2002

12. Morgenstern LB, Huber JC, Luna-Gonzales H, Saldin KR, Grotta JC, Shaw SG, Knudson L, Frankowski RF. Headache in the emergency department Headache 2001 Jun; 41(6): 537-41. doi: 10.1046/j.1526-4610.2001.041006537.x.

13. Filler L, Akhter M, Nimlos P. Evaluation and Management of the Emergency Department Headache.Semin Neurol. 2019 Feb; 39 (1): $20-26$.

14. Olesen J. IHS Classification: present and future. Cephalgia 1993; 13: 94

15. Rasmussen BK. Epidemiology of headache. Cephalalgia 2001 Sep; 21(7): 774-7. doi: 10.1177/033310240102100708.

16. Raskin NH. On the origin of head pain. Headache. New York, Churchill Livingstone 1988; 28: 254-7.

17. Rasmussen BK, Jensen R, Scrholl M. Epidemiology of headache in a genel population a prevelance study.J Clin Epidemiol 1991; 44: 1147-1157

18. Blumenthal HJ, Weisz MA, Kelly KM, Mayer RL, Blonsky J. Treatment of primary headache in the emergency department. Headache NovDec 2003; 43(10): 1026-31. doi: 10.1046/j.1526-4610.2003.03202.x.

19. Mills ML, Russo LS, Vines FS, Ross BA. High-yield criteria for urgent cranial computed tomography scans. Ann Emerg Med 1986 Oct; 15(10): 1167-72. doi: 10.1016/s0196-0644(86)80859-9.

20. Munoz-Ceron J, Marin-Careaga V, Peña L, Mutis J, Ortiz G. Headache at the emergency room: Etiologies, diagnostic usefulness of the ICHD 3 criteria, red and green flags. PLoS One. 2019 Jan 7; 14(1)

21. Rasmussen BK, Jensen R, Schroll M, Olesen J. Epidemiology of headache in a general population--a prevalence study. J Clin Epidemiol 1991; 44(11): 1147-57. doi: 10.1016/0895-4356(91)90147-2.

22. Beck S, Kinnear FB, Maree Kelly A, Chu KH, Sen Kuan W, Keijzers G, Body R, Karamercan MA, Klim S, Wijeratne T, Kamona S, Graham CA, Roberts T, Horner D, Laribi S; HEAD Study Group. Clinical presentation and assessment of older patients presenting with headache to emergency departments: A multicentre observational study. Australas J Ageing. 2021 Sep 27.

24. Wells S, Stiell IG, Vishnyakova E, Lun R, Nemnom MJ, Perry JJ. Optimal management strategies for primary headache in the emergency department. CJEM. 2021 Nov; 23(6): 802-811. doi: 10.1007/s43678-021-00173-0.

25. Rothrock JF, Diener HC. Headache secondary to cerebrovascular disease.Cephalalgia. 2021 Apr; 41(4): 479-492.

26. Lu J, Liu W, Zhao H. Headache in cerebrovascular diseases. Stroke Vasc Neurol. 2020 Jun; 5(2): 205-210.

Reviewer of the article: Assoc. prof. Dr. Mario Milkov, MD, PhD - Medical University - Varna

\author{
Correspondence to: \\ Onur Incealtin \\ Goztepe Prof. Dr. Suleyman Yalcin City Hospital, 34722, Kadikoy, Istanbul \\ e-mail: onur-incealtin@hotmail.com
}

